Fortschritte in der personalisierten Medizin, worunter zum Teil auch «Präzisionsmedizin» verstanden wird, und in der Genetik finden stetig Zugang in den Alltag der Gesundheitsversorgung. Dem Enthusiasmus, der in diesem Gebiet herrscht, ist aber, wie auch bei der digitalen Revolution, mit einer gesunden Skepsis zu begegnen. Letztlich darf die «condition humaine» nicht vergessen werden. Denn personalisierte Medizin ist mehr als die Sicht auf Moleküle und Gene. Sie gründet auch auf Empathie und der Beziehung zum Patienten. Der Besuch der Konferenz zum Thema - wo es u.a. um die Herausforderung geht, Präzisionsmedizin auf der Nano- und Mikroebene zu verbinden mit der individuellen Komplexität auf Meso- und Makroebene - lohnt sich.

Dr. med. Carlos Beat Quinto, Mitglied des FMH-Zentralvorstandes, Departementsverantwortlicher Public Health und Gesundheitsberufe

\title{
Personalisierte Gesundheit aus Public-Health-Perspektive
}

\section{Julia Dratva}

Dr. med., MD MPH, Präsidentin der Schweizerischen Gesellschaft der Fachärztinnen und -ärzte für Prävention und Gesundheitswesen

Niemals zuvor standen uns umfassendere Daten und Quellen zur Verfügung. Dadurch ergeben sich für die Gesundheitswissenschaften ganz neue Möglichkeiten. Die Auswertung von biochemischen und genetischen Daten, Diagnosen und Therapien sowie Informationen über Aktivitäten und Lebensstile erlauben uns, Diagnostik, Therapie und Prävention noch zielgerichteter zu entwickeln, um einerseits personalisierte Vorsorge und Behandlungen anbieten zu können und andererseits auf Bevölkerungsebene präventiv wirksame Massnahmen zu ergreifen. Derlei personalisierte Daten lassen sich wiederum mit einer Vielzahl von anderen Informationen verbinden, z.B. über Luftschadstoffe oder das Klima, und können wichtige neue Erkenntnisse liefern. So konnten Forschende in der Schweiz zum Beispiel nachweisen, dass die Luftbelastung am Wohnort einen Einfluss auf Diabetes-Erkrankungen hat. Voraussetzung dafür sind ein geregelter Zugang zu Daten sowie ein gemeinsames Verständnis von der Gesundheit der Bevölkerung.

An der Swiss Public Health Conference 2017 werden wir diese Themen aufnehmen. Wir gehen den Fragen nach, inwiefern personalisierte Medizin und Public Health miteinander vereinbar sind oder welche neuen Möglichkeiten sich für die öffentliche Gesundheit und die Prävention ergeben können. Auch ethische Aspekte werden berücksichtigt, denn neue Methoden werfen Fragen auf, etwa bezüglich Datenschutz. Schliesslich möchten wir den aufkommenden personalisierten Gesundheitsmarkt kritisch beleuchten und den gerechten Zugang zu personalisierter Medizin diskutieren.
Wir haben ein vielfältiges und praxisorientiertes Programm zusammengestellt. Es hält Anregungen und Ideen für Kooperationen bereit. Wir möchten gemeinsam mit Ihnen und internationalen Expertinnen und Experten diskutieren und freuen uns, Sie an der Swiss Public Health Conference 2017 in Basel begrüssen zu dürfen.

Die Konferenz wird gemeinsam von Public Health Schweiz (www.public-health.ch), der Swiss School of Public Health (www.ssphplus.ch) und Partnerorganisationen organisiert. Jedes Jahr übernimmt eine andere akademische Public-Health-Institution in der Schweiz die Rolle des Gastgebers und der wissenschaftlich-thematischen Leitung. Die diesjährige Swiss Public Health Conference zum Thema «Personalisierte Gesundheit aus Public-Health-Perspektive» wird gemeinsam mit dem Schweizerischen Tropen- und Public-Health-Institut (Swiss TPH, www.swisstph.ch) als Gastgeberin organisiert. Die Schweizerische Gesellschaft der Fachärztinnen und -ärzte für Prävention und Gesundheitswesen (www.sgpg.ch) wiederum verantwortet die ärztliche Weiter- und Fortbildung im Bereich Prävention und Gesundheitswesen in der Schweiz und engagiert sich für gute Rahmenbedingungen zugunsten der Gesundheit der Bevölkerung. In diesem Sinn empfiehlt die SGPG ihren Mitgliedern und allen an Public Health interessierten Kollegen und Kolleginnen die Konferenzteilnahme wärmstens.

http://conference.public-health.ch 